\title{
Atomic photoabsorption process controlled by static and oscillating magnetic fields
}

\author{
Takeshi Shirahama, ${ }^{1}$ Xiao-Min Tong, ${ }^{1,2, *}$ Ken-ichi Hino, ${ }^{3}$ and Nobuyuki Toshima ${ }^{1}$ \\ ${ }^{1}$ Institute of Materials Science, University of Tsukuba, 1-1-1 Tennodai, Tsukuba, Ibaraki 305-8573, Japan \\ ${ }^{2}$ Center for Computational Sciences, University of Tsukuba, 1-1-1 Tennodai, Tsukuba, Ibaraki 305-8577, Japan \\ ${ }^{3}$ Doctoral Program in Frontier Science, Graduate School of Pure and Applied Sciences, University of Tsukuba, 1-1-1 Tennodai, Tsukuba, \\ Ibaraki 305-8573, Japan
}

(Received 1 September 2009; published 23 October 2009)

\begin{abstract}
We present a theoretical method to study atomic photoabsorption processes in a temporally periodic external field. The time development of the atomic dipole moment is propagated by numerical time propagation, and the photoabsorption cross sections is obtained directly from the Fourier transform of the autocorrelation function without summing up over the Floquet-Fourier components. As an example, we study the photoabsorption spectra of hydrogen atoms exposed in a mixture of static and periodically oscillating magnetic fields for the transition from $1 s \rightarrow 2 p$. The atomic energy structure and photoabsorption spectra show interesting dependence on the strength of the oscillating magnetic field. Tuning the combination of the magnetic field strengths, we can control the photoabsorption process. The proposed method is general and can be applied to processes in which the number of relevant high harmonic components of the Fourier expansion is so large that the conventional Floquet method is intractable.
\end{abstract}

DOI: $10.1103 /$ PhysRevA.80.043414

PACS number(s): 32.80.Qk, 32.60.+i, 31.15.-p

\section{INTRODUCTION}

Photoabsorption has been an important process to study the atomic structures since the advent of the quantum mechanics. Each atom has a unique energy structure and we can identify the constitutions of a material from the photoabsorption spectra. For most of atoms, the energy structures and absorption spectra are known and were tabulated many years ago [1]. The interest of recent research has turned to how to control the atomic energy structures and the dynamic processes. One can control the atomic process by applying static electric fields [2,3], static magnetic fields [4,5], and crossed static electric and magnetic fields [6,7]. One can also control the atomic process by applying an oscillating electric (or magnetic) field $[8,9]$. The infrared-laser-assisted photoionization [10-12] is one of the recent hot topics in atomic physics and it is another example to control the photoabsorption by a periodic electric field.

A conventional theoretical method to study atomic dynamics in a temporally periodic field is based on the Floquet theory [13]. In this theory, we transform the time-dependent Schrödinger equation into a set of coupled time-independent equations and solve it as an eigenvalue problem by diagonalization. The photoabsorption cross sections can be obtained from the transition matrix elements between the ground state and the excited Floquet states. The numerical burden increases cubically as the number of the coupled equations increases. This rapid increase of the computational load limits the applicability of the Floquet method. A way to circumvent the numerical difficulty involved in the Floquet method is to calculate the wave function directly using a timepropagation method [13]. The Floquet wave functions are obtained by the Fourier transformation of the time-dependent numerical wave functions, but this procedure also needs

\footnotetext{
*tong.xiaomin.ga@u.tsukuba.ac.jp
}

huge computational resources if all the wave functions are needed.

In this paper we develop a theoretical method to study atomic photoabsorption processes in temporally periodic external fields without calculating the Floquet wave function explicitly. We prepare the initial state as the product of the dipole operator and the ground-state wave function. We then propagate the initial wave function under the total Hamiltonian and calculate the autocorrelation function or a modified autocorrelation function. Finally, we obtain the photoabsorption cross section from a Fourier transform of the autocorrelation function. We take the photoabsorption process of hydrogen atoms from $1 s \rightarrow 2 p$ in the mixture of static and temporally periodic magnetic fields as an example in order to demonstrate the validity and usefulness of the method since the problem can be also treated by the conventional Floquet theory. We formulate our theoretical method in Sec. II and present the results in Sec. III.

\section{THEORETICAL METHOD}

We study the dynamic process of an atom in a temporally periodic external field. The Schrödinger equation to be solved is written as (atomic units $m=\hbar=e=1$ are used throughout unless otherwise stated)

$$
i \frac{\partial}{\partial t} \Psi(t)=H(t) \Psi(t)=\left[H_{0}+V(t)\right] \Psi(t),
$$

where $H_{0}$ is the atomic Hamiltonian, $V(t)=V(t+T)$ the external field with a period $T$, and $\Psi(t)$ the time-dependent wave function of the total system. According to the Floquet theory [13], a solution of the above equation can be expanded as

$$
\Psi_{\alpha}(t)=e^{-i \epsilon_{\alpha} t} \sum_{n} e^{-i n \omega t} \phi_{\alpha n},
$$

with $\omega=2 \pi / T$. Here $\epsilon_{\alpha}$ is the quasienergy and $\phi_{\alpha n}$ is the associated time-independent wave function of the $n$th Fourier 
component (sideband). Expanding the potential $V(t)$ as a Fourier series,

$$
V(t)=\sum_{n} e^{-i n \omega t} V_{n}
$$

Equation (1) is converted to coupled equations,

$$
\left(\epsilon_{\alpha}+n \omega-H_{0}\right) \phi_{\alpha n}=\sum_{k=-\infty}^{\infty} V_{n-k} \phi_{\alpha k} .
$$

When the periodic external field changes the energy structures of excited states but the ground state stays perturbed little, the photoabsorption cross section of a probe light with an energy $\omega_{p}$ for the transition from the ground state $\phi_{g}$ to excited Floquet states is expressed as

$$
\sigma\left(\omega_{p}\right)=\frac{4 \pi \omega_{p}}{c} \operatorname{Im} \sum_{\alpha n} \frac{\left\langle\phi_{g}|\hat{d}| \phi_{\alpha n}\right\rangle\left\langle\phi_{\alpha n}|\hat{d}| \phi_{g}\right\rangle}{\left(\epsilon_{\alpha}+n \omega\right)-\left(\epsilon_{g}+\omega_{p}\right)-i \eta} .
$$

$c$ is the velocity of light, $\hat{d}$ the dipole operator and $\eta$ a positive infinitesimal number to ensure the adiabatic switching on of the probe field. Im denotes the imaginary part of the quantity. For systems in which the number of involved physical states is not large, the quasienergy $\left\{\epsilon_{\alpha}\right\}$ and the timeindependent wave function $\left\{\phi_{\alpha n}\right\}$ can be obtained by solving the Floquet coupled equations (4) by diagonalization. The convergence for the sum over $n$ is, however, rather slow in general and the coupled equations become intractable as the number of the Floquet states increases. Another way to solve Eq. (1) is to use a time-dependent method proposed by Telnov and Chu [14]. Their method, however, has to propagate all the atomic wave functions over one optical cycle. All the quasieigenenergies and wave functions at time $t=0$ are computed by diagonalization of the one-period time-evolution operator.

Here we propose an alternative way in which we can obtain the dynamic information without calculating the Floquet wave functions. We solve at first Eq. (1) numerically under the initial condition defined as $|\Psi(t=-\infty)\rangle=\hat{d}\left|\phi_{g}\right\rangle$ to obtain the time-dependent wave function $|\Psi(t)\rangle$ and then calculate a modified autocorrelation function defined as

$$
C(t)=\langle\Psi(t=-\infty) \mid \Psi(t)\rangle=\left\langle\phi_{g}|\hat{d}| \Psi(t)\right\rangle,
$$

and its Fourier transform

$$
A\left(\omega_{p}\right)=\int_{-\infty}^{\infty} C(t) e^{i\left(\epsilon_{g}+\omega_{p}\right) t} d t .
$$

These integrals are evaluated at each time step without storing the numerical values of the previous time-dependent wave functions. The photoabsorption cross section is obtained as

$$
\sigma\left(\omega_{p}\right)=\frac{2 \pi \omega_{p}}{c} \frac{A^{*}\left(\omega_{p}\right) A\left(\omega_{p}\right)}{\sum_{n=-\infty}^{\infty} A\left(\omega_{p}+n \omega\right)} .
$$

This is an extension of the formula used in the study of multiphoton ionization $[15,16]$ and photoabsorption of hy- drogen atoms in the crossed static electric and magnetic fields [6]. When the time-dependent field strength goes to zero, there is only one nonzero component in the summation of the denominator of Eq. (8) for a specified $\omega_{p}$ and the formula reduces to the simpler expression for the static field case.

We prove the equivalence of Eqs. (5) and (8) in the following. Since the solutions $\left\{\Psi_{\alpha}\right\}$ of Eq. (1) form a complete basis set, we can expand the time-dependent wave function $\Psi(t)$ as

$$
\Psi(t)=\sum_{\alpha} D_{\alpha} \Psi_{\alpha}(t)
$$

with

$$
D_{\alpha}=\sum_{n}\left\langle\phi_{\alpha n}|\hat{d}| \phi_{g}\right\rangle
$$

The autocorrelation function can be expressed formally as

$$
C(t)=\sum_{\alpha, n} D_{\alpha}\left\langle\psi_{g}|\hat{d}| \phi_{\alpha n}\right\rangle e^{-i\left(\epsilon_{\alpha}+n \omega\right) t},
$$

and its Fourier transform

$$
A\left(\omega_{p}\right)=2 \pi \sum_{\alpha, n} D_{\alpha}\left\langle\psi_{g}|\hat{d}| \phi_{\alpha n}\right\rangle \delta\left(\epsilon_{g}+\omega_{p}-\epsilon_{\alpha}-n \omega\right) .
$$

Using the delta function property, if we focus on a given Floquet state $\alpha$, i.e., $\bar{\omega}_{p}=\bmod \left(\epsilon_{g}+\omega_{p}, \omega\right)=\epsilon_{\alpha}$, we obtain

$$
\sum_{n} A\left(\bar{\omega}_{p}+n \omega\right)=\sum_{n} 2 \pi D_{\alpha}\left\langle\psi_{g}|\hat{d}| \psi_{\alpha n}\right\rangle=2 \pi D_{\alpha} D_{\alpha}^{*} .
$$

Since

$$
\operatorname{Im} \lim _{\eta \rightarrow 0} \frac{1}{x-i \eta}=\pi \delta(x),
$$

both Eqs. (5) and (8) reach the same expression,

$$
\begin{aligned}
\sigma\left(\omega_{p}\right)= & \frac{4 \pi^{2} \omega_{p}}{c} \sum_{n}\left\langle\phi_{g}|\hat{d}| \phi_{\alpha n}\right\rangle\left\langle\phi_{\alpha n}|\hat{d}| \phi_{g}\right\rangle \\
& \times \delta\left(\epsilon_{g}+\omega_{p}-\epsilon_{\alpha}-n \omega\right) .
\end{aligned}
$$

The $\delta$ function represents the energy conservation. When we derive the above equations, we assume that there is no degenerate state and this assumption is valid for the example we present in this paper.

\section{RESULTS AND DISCUSSION}

To test the validity of the method, we investigate the photoabsorption of hydrogen atoms for the transition of $1 s$ $\rightarrow 2 p$ controlled by the combined static and periodic magnetic fields. We choose a static magnetic field $B_{x}$ along the $x$ direction, and another static field $B_{z}$ and a periodic magnetic field $B_{0} \cos \omega t$ with a frequency $\omega$ along the $z$ direction. The combination of the static and oscillating magnetic fields is chosen in the analogy to the band structure of semiconductor superlattices $[9,17]$. The Hamiltonian of the system in the Coulomb gauge is read as 


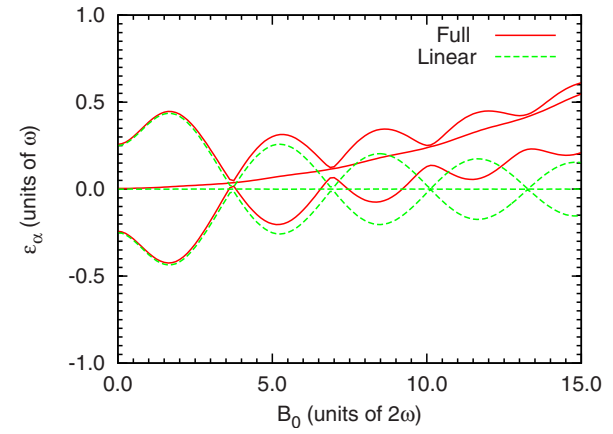

FIG. 1. (Color online) The quasienergies of hydrogen $2 p$ states in the combined static and period magnetic fields with the linear (dashed curve) and the full (solid curve) Zeeman terms.

$$
H=-\frac{\nabla^{2}}{2}-\frac{1}{r}+\frac{1}{2} \vec{L} \cdot \vec{B}+\frac{1}{8}(\vec{r} \times \vec{B})^{2},
$$

with $\vec{B}=\left(B_{x}, 0, B_{z}+B_{0} \cos \omega t\right)$ and $\vec{L}$ the angular momentum operator. In writing the above equation, we ignore the spin contributions. The parameters used in the calculations are $B_{x}=6.0 \times 10^{-4}, B_{z}=8.0 \times 10^{-4}$ and $\omega=4.0 \times 10^{-4}$. We also assume that the probe light is polarized along the $z$ direction. Thus the initial state is expressed as $|\Psi(t=-\infty)\rangle=z|1 s\rangle$ where $z$ is the $z$ coordinate of the electron. We use relatively weak magnetic fields and low frequencies $\omega$ so that $2 p$ states are dominantly coupled. There are three $2 p$ states with the magnetic quantum numbers $m=-1,0,1$. Using the three states as a basis set, the Hamiltonian can be represented as a $3 \times 3$ matrix.

There are various ways to propagate the time-dependent wave function in Eq. (1) depending on the physics involved. For the present simple example, we propagate the wave function by the numerical scheme of Crank-Nikolson as

$$
\begin{aligned}
\Psi(t+\Delta t) \simeq & {\left[1+i H\left(t+\frac{3}{4} \Delta t\right) \Delta t / 2\right]^{-1} } \\
& \times\left[1-i H\left(t+\frac{1}{2} \Delta t\right) \Delta t / 2\right] \Psi(t) .
\end{aligned}
$$

The choice of the numerical scheme for integration on time is not essential as far as the differential equation is solved with sufficient accuracy.

If we use the Hamiltonian matrix $H$ in the propagator and diagonalize the propagator after integrating over one period, we obtain the Floquet matrix which give the quasienergies after diagonalization. The calculated quasienergies are in good agreement with the values obtained by the conventional Floquet method for more than ten digits. The ground state $\phi_{g}$ is perturbed little by these magnetic fields since the linear Zeeman term vanishes and the quadratic Zeeman term is negligible for the present case.

Figure 1 shows the calculated quasienergies as a function of $B_{0}$. We choose the field-free $2 p$ energy as zero in the scale. Based on the Floquet theory, there appear many replicas for each quasienergy shifted by multiples of $\omega$ and we only show the parent band (quasienergies) structure. The $2 p$ energies split into three levels as expected. If we take into ac-

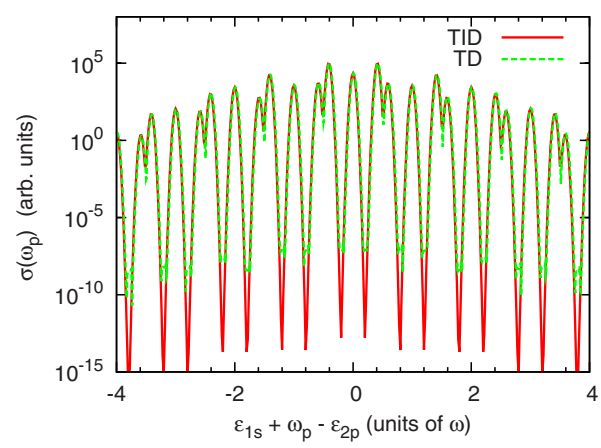

FIG. 2. (Color online) The photoabsorption probabilities of hydrogen atoms in the combined static and periodic magnetic fields calculated by the present time-dependent method (TD, dashed curve) and the conventional Floquet method (TID, solid curve).

count only the linear Zeeman term, one of the energy levels is independent of $B_{0}$ and the other two levels oscillate with damping amplitudes for increasing $B_{0}$. Addition of the quadratic Zeeman effect shifts the energies to the higher energy side as a result of the confinement of the electron by the magnetic field.

The photoabsorption cross sections are shown in Fig. 2 for $B_{0}=1.0 \times 10^{-3}$. The cross sections of the conventional Floquet theory are also shown for comparison. In the practical calculations we cannot propagate the wave function from $t=-\infty$ to $t=\infty$, and hence we propagate the wave function from $t=0$ to $t=128 T$ for the period $T$ of the oscillating magnetic field. To suppress the inaccuracy induced by the finite time propagation, we multiply a filter function $f(t)$ $=\exp \left[-(t-64 T)^{2} /\left(100 T^{2}\right)\right]$ to the autocorrelation function
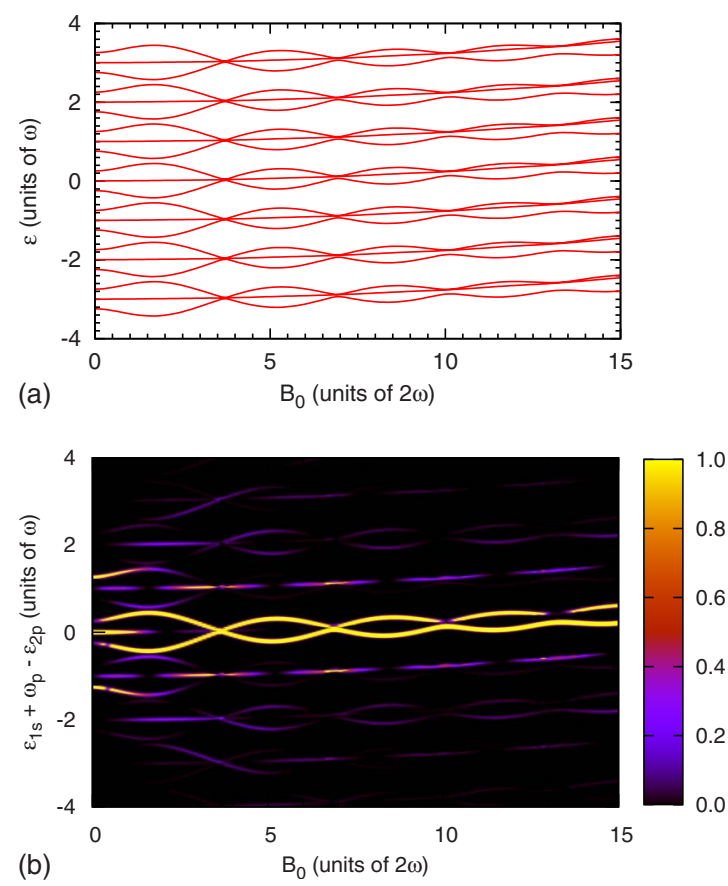

FIG. 3. (Color online) The energy structures (upper panel) and the photoabsorption cross section (lower panel) of hydrogen atoms in the combined static and periodic magnetic fields calculated by the present time-dependent method. 
$C(t)$. This is equivalent to the adiabatic switch on and off of the probe light. To make the comparison with the conventional Floquet results meaningful, we also convolute the photoabsorption cross sections of Eq. (5) with the same Gaussian type energy resolution. We see the two calculated results are also identical for more than ten digits.

The photoabsorption cross sections show many side-band structures and, although the energy structures are similar to each other, the magnitude of the photoabsorption cross sections differs significantly for different bands. Figure 2 shows the photoabsorption cross sections for a fixed $B_{0}$. If we tune $B_{0}$, we can control the photoabsorption spectra as shown in Fig. 3. We also plot the energy levels of the sidebands in the upper panel of Fig. 3 for comparison. In the Floquet theory, the energy structures of the parent band and the sidebands are the same. The energy levels of three $2 p$ states are repeated periodically with the interval $\omega$. For the photoabsorption process, however, the transition strengths to the different bands vary significantly. When $B_{0}=0$, no periodic magnetic field is present and there exist only the three states of the parent band split by the static magnetic field. As we switch on the oscillating magnetic field and increase the field strength gradually, the photoabsorption tends to concentrate to the parent bands after side-band absorption shows up at first. The photoabsorption is dominated by the two levels of the parent band for $B_{0}>10$. This tendency is not intuitively evident since the increase of the oscillating field strength is expected to enhance the periodic nature of the dynamics. Tuning the strength of the applied periodic magnetic field we can control the photoabsorption process at a specified photon energy.

So far we assumed that the ground state is not perturbed by the external field. The numerical method is still valid for a strong periodic field if we replace the ground state with a perturbed initial state in the formulas.

To summarize, we proposed a theoretical method to calculate the photoabsorption cross section in a temporally periodic external field. Taking the photoabsorption of hydrogen atoms as an example, we showed that the photoabsorption process can be controlled by tuning the applied periodic magnetic field strength. The method can be applied to a general case of atomic systems in a periodic external field.

\section{ACKNOWLEDGMENTS}

This research was supported by Grant-in-Aid for Scientific Research (C) from the Japan Society for the Promotion of Science.
[1] C. E. Moore, Atomic Energy Levels (National Bureau of Standards, Washington, D.C., 1971)

[2] J. R. Harries, J. P. Sullivan, J. B. Sternberg, S. Obara, T. Suzuki, P. Hammond, J. Bozek, N. Berrah, M. Halka, and Y. Azuma, Phys. Rev. Lett. 90, 133002 (2003).

[3] X. M. Tong and C. D. Lin, Phys. Rev. Lett. 92, 223003 (2004).

[4] A. Holle, G. Wiebusch, J. Main, G. Z. K. H. Welge, G. Wunner, T. Ertl, and H. Ruder, Z. Phys. D: At., Mol. Clusters 5, 279 (1987).

[5] S. I. Chu and X. M. Tong, Chem. Phys. Lett. 294, 31 (1998).

[6] X. M. Tong and Shih-I Chu, Phys. Rev. A 61, 031401(R) (2000).

[7] G. Wiebusch, J. Main, K. Kruger, H. Rottke, A. Holle, and K. H. Welge, Phys. Rev. Lett. 62, 2821 (1989).

[8] S. Haroche, C. Cohen-Tannoudji, C. Audoin, and J. P. Scher- mann, Phys. Rev. Lett. 24, 861 (1970).

[9] M. Holthaus and D. W. Hone, Philos. Mag. B 74, 105 (1996).

[10] M. P. Hertlein, H. Adaniya, J. Amini, C. Bressler, B. Feinberg, M. Kaiser, N. Neumann, M. H. Prior, and A. Belkacem, Phys. Rev. A 73, 062715 (2006).

[11] S. H. Southworth et al., Phys. Rev. A 76, 043421 (2007).

[12] P. Johnsson, J. Mauritsson, T. Remetter, A. L'Huillier, and K. J. Schafer, Phys. Rev. Lett. 99, 233001 (2007).

[13] S. I. Chu and D. A. Telnov, Phys. Rep. 390, 1 (2004).

[14] D. A. Telnov and S.-I. Chu, J. Phys. B 37, 1489 (2004).

[15] E. J. Heller, J. Chem. Phys. 68, 2066 (1978).

[16] G. P. Arrighini, F. Biondi, and C. Guidotti, J. Phys. B 19, 1297 (1986).

[17] K. Yashima, K. I. Hino, and N. Toshima, Phys. Rev. B 68, 235325 (2003). 